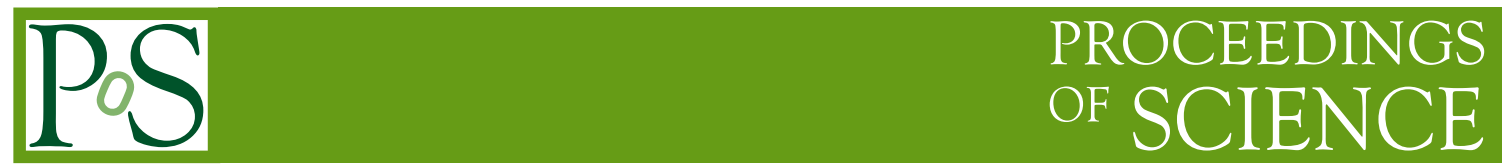

\title{
Experimental measurement of Heavy Flavors and jets
}

\author{
Meena Meena ${ }^{a, b}$ \\ a Panjab University, \\ Chandigarh, India \\ ${ }^{b}$ On behalf of the ALICE, ATLAS, CMS, and LHCb Collaborations \\ E-mail: meena.meena@cern.ch
}

Recent results of the heavy flavors and jet production in proton-proton collisions using the data collected by the ALICE, ATLAS, CMS, and LHCb detector at the Large Hadron Collider are presented. These measurements are crucial for precise tests of electroweak and next-to-leadingorder pQCD calculations, improving the understanding of the proton structure. The measured results are compared with theoretical predictions from several Monte Carlo event generators using different parton shower simulations which provide valuable inputs for tuning of Monte Carlo event generators.

The Ninth Annual Conference on Large Hadron Collider Physics - LHCP2021

7-12 June 2021

Online 


\section{Introduction}

In this document, an overview of recent results on heavy flavors (HF) and jet productions and their comparison with various theoretical predictions are presented in the proton-proton collision with the ALICE [1], ATLAS [2], CMS [3], and LHCb [4] experiments.

\section{Measurement of the $\mathrm{HF}$ dijet and $\mathrm{Z}+\mathrm{HF}$ jets cross section at the center-of-mass energy $(\sqrt{s})$ of $13 \mathrm{TeV}$}

The first differential and integrated cross section measurements of inclusive $b \bar{b}$ - and $c \bar{c}$-dijet [5] production are performed with the LHCb detector. The phase space regions are defined by requiring the two jets originating from the two $\mathrm{b}$ or $\mathrm{c}$ quarks with transverse momentum $\left(p_{T}\right)$ greater than 20 $\mathrm{GeV}$, pseudorapidity $(\eta)$ in the range $2.2<\eta<4.2$, and with a difference in the azimuthal angle between the two jets greater than 1.5. The integrated $b \bar{b}$ - and $c \bar{c}$-dijet cross sections are measured to be $53.0 \pm \leq 0.1$ (stat) \pm 9.5 (stat+syst) \pm 2.1 (lumi) nb, and $73 \pm \leq 0.1$ (stat) \pm 16.1 (stat+syst) \pm 2.9 (lumi) $\mathrm{nb}$ and the ratio between $c \bar{c}$ - and $b \bar{b}$-dijet cross section is $1.37 \pm \leq 0.01$ (stat) \pm 0.27 (stat+syst). The differential cross section as a function of leading jet $p_{T}$ is shown in Figure 1 (left).

The differential and integrated cross section measurements of $Z(\rightarrow l l)+\geq 1 \mathrm{c}$ jet [6], where $l l=e^{+} e^{-}$or $\mu^{-} \mu^{+}$and cross section ratios of $\mathrm{Z}+\mathrm{b}$ jet and $\mathrm{Z}+\mathrm{c}$ jet w.r.t $\mathrm{Z}+$ jet $(\mathrm{R}(\mathrm{b} / \mathrm{j}), \mathrm{R}(\mathrm{c} / \mathrm{j}))$ and $\mathrm{Z}+\mathrm{c}$ jet w.r.t $\mathrm{Z}+\mathrm{b}$ jet $(\mathrm{R}(\mathrm{c} / \mathrm{b}))$ [7] are performed with the CMS detector. The fiducial phase space regions are defined by selecting $\mathrm{Z}$ boson with at least one inclusive or $\mathrm{b}$ jet or $\mathrm{c}$ jet selected with $p_{T}>30 \mathrm{GeV}$ and $|\eta|<2.4$. The total fiducial measured cross section of $\mathrm{Z}+\mathrm{c}$ jet to be $405.4 \pm 5.6$ (stat) $\pm 24.3(\exp ) \pm 3.7$ (theo) $\mathrm{pb}$. The measured integrated cross section ratio values are $\mathrm{R}(\mathrm{c} / \mathrm{j})$ $=0.102 \pm 0.002$ (stat) \pm 0.009 (syst), $\mathrm{R}(\mathrm{b} / \mathrm{j})=0.0633 \pm 0.0004$ (stat) \pm 0.0015 (syst), and R (c/b) $=1.62 \pm 0.03$ (stat) \pm 0.15 (syst). The predictions for $\mathrm{Z}+\mathrm{b}$ and $\mathrm{Z}+\mathrm{c}$ jet production can be derived in either a 4-flavor number scheme (4FNS) or a 5-flavor number scheme (5FNS). In 5FNS, b quark density is allowed in the initial state via $a b$ quark parton distribution functions (PDFs) of proton but not in 4FNS. The measured integrated cross section of $\mathrm{Z}+\mathrm{c}$ jet, $\mathrm{R}(\mathrm{c} / \mathrm{j})$, and $\mathrm{R}(\mathrm{b} / \mathrm{j}), b \bar{b}$ - and $c \bar{c}$-dijet are overestimated by MG5_aMC next-to-leading order (NLO) 5FNS, except $\mathrm{R}(\mathrm{c} / \mathrm{b})$. The $\mathrm{Z}+\mathrm{c}$ jet, $\mathrm{R}(\mathrm{c} / \mathrm{j})$, and $\mathrm{R}(\mathrm{b} / \mathrm{j})$ are well described by the MG5_aMC LO 5FNS and $b \bar{b}$ - and $c \bar{c}$-dijet by PYTHIA8 LO but Z+c jet overestimated by the SHERPA NLO 5FNS. The R (c/j) and R (c/b) are underestimated and $R(b / j)$ is overestimated by the MCFM predictions at NLO and LO.

A measurement of the inclusive and differential production cross sections of a $\mathrm{Z}(\rightarrow l l)+\geq$ $1 \mathrm{~b}$ jet and $\mathrm{Z}(\rightarrow l l)+\geq 2 \mathrm{~b}$ jets [8] are performed with the ATLAS detector. The measured inclusive cross sections for $\mathrm{Z}+\geq 1 \mathrm{~b}$ jet and $\mathrm{Z}+\geq 2 \mathrm{~b}$ jets for jet $\mathrm{p}_{T}>20 \mathrm{GeV}$ and rapidity $|y|<2.5$ as shown in Figure 1 (middle and right), are $10.90 \pm 0.03$ (stat) \pm 1.08 (syst) \pm 0.25 (lumi) pb and $1.32 \pm 0.01$ (stat) \pm 0.21 (syst) \pm 0.04 (lumi) pb, respectively. The 5FNS simulations adequately predict the inclusive cross sections for both $Z+\geq 1$ b jet $\& Z+\geq 2$ b jets. All 4FNS MC predictions are systematically lower than data for $Z+\geq 1$ b jet but agree well for $Z+\geq 2$ b jets except Alpgen+Py6 LO 4FNS which underestimates the data. The use of the NNPDF3.0lo PDF set in Alpgen predictions gives better agreement with data because of a higher acceptance in the fiducial region. The SHERPA fusing 4FNS+5FNS at NLO simulation agrees with Sherpa 5FNS NLO, showing that the effects of merging are minor. 

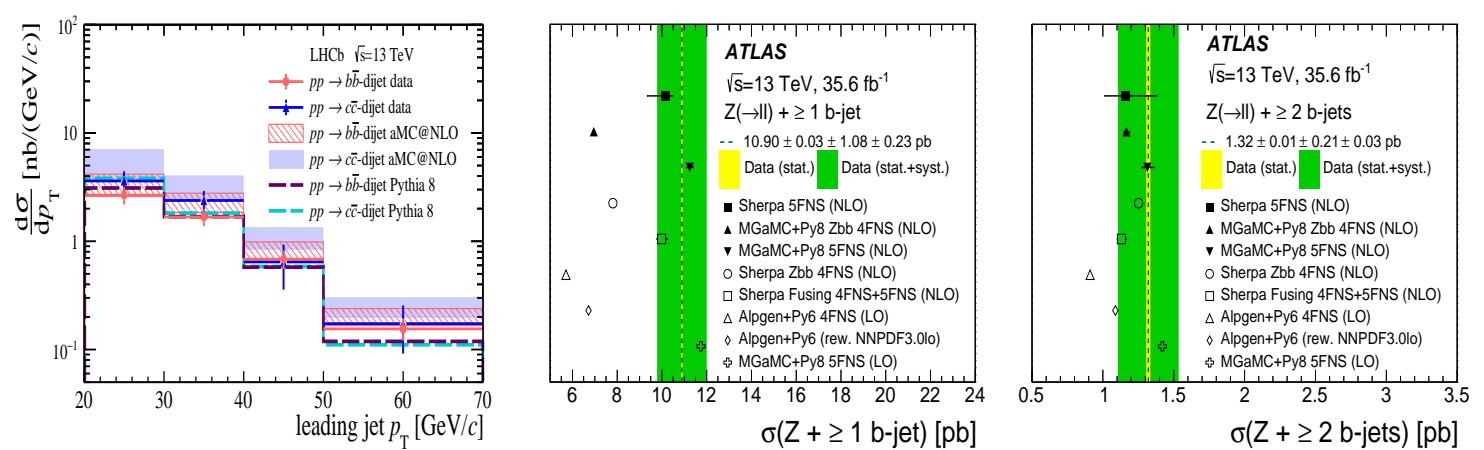

Figure 1: Differential $b \bar{b}$ - and $c \bar{c}$-dijet cross section as a function of leading jet $p_{T}$ and error bars represent the total uncertainties [5] (left). The integrated cross sections for $Z+\geq 1 b$ jet (middle) and $Z+\geq 2 b$ jets (right) [8].

\section{Study of $J / \psi$ and $\psi(2 s)$ meson production inside jets}

The first study of prompt $J / \psi \rightarrow \mu^{+} \mu^{-}$mesons contained in jets produced in the gluon fragmentation dominated central rapidity region $(\mid \eta($ jet $) \mid<1)$ with $\mathrm{p}_{T}($ jet $)>25 \mathrm{GeV}$, is performed based on the fragmenting jet function (FJF) approach with the CMS detector at $\sqrt{s}=8 \mathrm{TeV}$ [9]. The FJF analysis uses the non-relativistic quantum chromodynamics (NRQCD) approach to compute the cross section for the formation of a $J / \psi$ meson from the $c \bar{c}$ system. The shape of measured differential cross section for $J / \psi$ meson production as a jet constituent (z) is compared to the FJF prediction, using three different long-distance matrix element (LDME) parameter sets the Bodwin, Chung, Kim, and Lee (BCKL), Butenschoen, and Kniehl (BK), and Chao. The data agree best with FJF BCKL calculation that use an LDME parameter set in which prompt $J / \psi$ mesons are predicted to be unpolarized for all three $\mathrm{z}$ ranges $(0.40-0.45,0.50-0.55$, and $0.60-0.65)$. In contrast, the FJF BK and Chao predictions disagree with the data for all three $\mathrm{z}$ ranges. The FJF BCKL not only describes the production of high- $p_{T} J / \psi$ mesons as constituents of jets but also predicts small $J / \psi$ meson polarization.

The first measurement $J / \psi \rightarrow \mu^{+} \mu^{-}$mesons produced in jets promptly and in b-hadron decay is studied in the forward region $(2.5<\eta$ (jet) $<4.0)$ with $p_{T}$ (jet $)>20 \mathrm{GeV}$ with the $\mathrm{LHCb}$ detector at $\sqrt{s}=13 \mathrm{TeV}$ [10]. Fraction of $J / \psi$ mesons produced in b-hadron decays is in the range 20-60\%, depending on $\mathrm{z}(J / \psi)$ and jet $\mathrm{p}_{T}$. The observed normalized cross section distribution for $J / \psi$ mesons produced in b-hadron decays is consistent with the PYTHIA8 prediction; however, the prompt- $J / \psi$ results do not agree with the LO NRQCD-based predictions as implemented in PYTHIA8 as shown in Figure 2 (left). At small $\mathrm{z}(J / \psi)$, PYTHIA8 predicts that most of the jet arises from a parton-parton scatter other than the one that produced the $J / \psi$ meson.

The measurements of the prompt and non-prompt double differential cross sections of $J / \psi$ and $\psi(2 s)$ mesons with jet $p_{T}$ between 60 and $360 \mathrm{GeV}$ for $J / \psi$ and 60 and $140 \mathrm{GeV}$ for $\psi(2 s)$ and $|y|<2$ are performed using data recorded by the ATLAS detector at $\sqrt{s}=13 \mathrm{TeV}$ between 2015 and 2018 [11]. Furthermore, the measurements of the non-prompt fractions of $\psi(2 s)$ and $J / \psi$, and prompt and non-prompt production ratios of $\psi(2 s)$ to $J / \psi$ are presented. In $J / \psi$ and $\psi(2 s)$ both cases, $p_{T}$ range goes well beyond the values reached so far, which may help discriminate various 
theoretical models. The results show similar $p_{T}$-dependence for prompt and non-prompt double differential cross sections, with non-prompt fractions close to constant $\sim 70 \%$ for both $J / \psi$ and $\psi(2 s)$. The prediction with the FONLL model is consistent with data for non-prompt production at the low end of the $p_{T}$ range but exceeds the experimental values at large $p_{T}$ as shown in Figure 2 (middle).

\section{Groomed jet substructure measurements of charm jets tagged with $D^{0}$ mesons}

This is the first measurement of groomed jet substructure observables for jets containing a fully reconstructed $\mathrm{D}^{0}$ meson at $\sqrt{s}=13 \mathrm{TeV}$ performed with the charged jet in $15 \leq \mathrm{p}_{T}<30 \mathrm{GeV}$ range with the ALICE detector. The Soft Drop grooming procedure is used to unwound the reclustered jets, starting from the final step until a fully reconstructed $\mathrm{D}^{0}$ meson candidate is found or all splittings have been exhausted. The momentum fraction of groomed two-prong jet substructure $\left(\mathrm{z}_{g}\right)$, the aperture angle between these prongs $\left(\mathrm{R}_{g}\right)$, and the number of splittings satisfying the grooming condition $\left(\mathrm{n}_{S D}\right)$ are the jet substructure observables. In particular, $\mathrm{n}_{S D}$ shifted to a smaller value for the $\mathrm{D}^{0}$-tagged jets shows significant sensitivity to differences in the $\mathrm{D}^{0}$-tagged and inclusive-jets samples as shown in Figure 2 (right). This indicates that the fragmentation of the $\mathrm{c}$ quark is hard throughout the showering procedure. The PYTHIA8 simulations with Monash tune calculation describing the data qualitatively well for all measured observables.
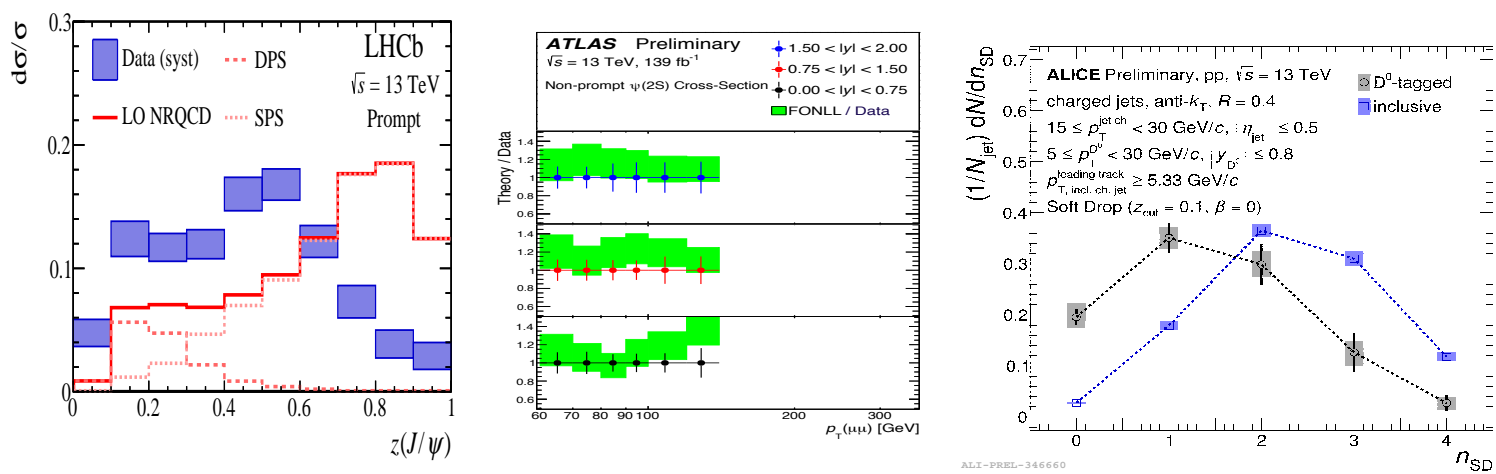

Figure 2: Normalized $z(J / \psi)$ distributions for $J / \psi$ mesons produced in b-hadron decay [10] (left). The ratios of the FONLL prediction to the measured differential cross sections are shown for non-prompt $\psi(2 s)$ mesons [11] (middle). The measured $n_{S D}$ distributions, corrected to particle level, of prompt $\mathrm{D}^{0}$-tagged and inclusive jets are shown (right) [12] (right).

\section{Summary}

The ALICE, ATLAS, CMS, and LHCb experiments have a rich program on the measurements related to heavy flavors and jet. Here, we presented an overview of many recent measurements which are sensitive to different theoretical approaches based on perturbative and non-perturbative methods and $b$ and $c$ quark PDFs in different kinematic regimes of jets production. 


\section{References}

[1] ALICE Collaboration, The ALICE experiment at the CERN LHC, JINST 3 (2008) S08002.

[2] ATLAS Collaboration, The ATLAS Experiment at the CERN Large Hadron Collider, JINST 3 (2008) S08003

[3] CMS Collaboration, The CMS Experiment at the CERN LHC, JINST 3 (2008) S08004

[4] LHCb Collaboration, The LHCb Detector at the LHC, JINST 3 (2008) S08005

[5] LHCb Collaboration, Measurement of differential $b \bar{b}$ - and $c \bar{c}$-dijet cross-sections in the forward region of pp collisions at $\sqrt{s}=13 \mathrm{TeV}$, JHEP 02 (2021) 023

[6] CMS Collaboration, Measurement of differential cross sections for $Z$ bosons produced in association with charm jets in pp collisions at $\sqrt{s}=13 \mathrm{TeV}$, JHEP 04 (2021)

[7] CMS Collaboration, Measurement of the associated production of a $Z$ boson with charm or bottom quark jets in proton-proton collisions at $\sqrt{s}=13 \mathrm{TeV}$, Phys. Rev. D 102 (2020)

[8] ATLAS Collaboration, Measurements of the production cross-section for a $Z$ boson in association with b-jets in proton-proton collisions at $\sqrt{s}=13 \mathrm{TeV}$ with the ATLAS detector, JHEP 07 (2020) 044

[9] CMS Collaboration, Study of $J / \psi$ meson production inside jets in pp collisions at $\sqrt{s}=8 \mathrm{TeV}$, Phys. Lett. B 804 (2020) 135409

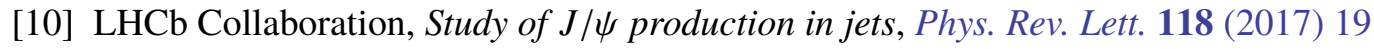

[11] ATLAS Collaboration, Measurement of the production cross-section of $J / \psi$ and $\psi(2 \mathrm{~S})$ mesons at high transverse momentum in pp collisions at $\sqrt{s}=13 \mathrm{TeV}$ with the ATLAS detector, ATLASCONF-2019-047

[12] ALICE Collaboration, Groomed jet substructure measurements of charm jets tagged with $\mathrm{D}^{0}$ mesons in pp collisions at $\sqrt{s}=13 \mathrm{TeV}$, ALICE-PUBLIC-2020-002 